IJMMS 29:10 (2002) 573-577

PII. S0161171202007822

http://ijmms.hindawi.com

(c) Hindawi Publishing Corp.

\title{
A CHARACTERIZATION OF HARMONIC FOLIATIONS BY THE VOLUME PRESERVING PROPERTY OF THE NORMAL GEODESIC FLOW
}

\author{
HOBUM KIM
}

Received 22 July 2001

\begin{abstract}
We prove that a Riemannian foliation with the flat normal connection on a Riemannian manifold is harmonic if and only if the geodesic flow on the normal bundle preserves the Riemannian volume form of the canonical metric defined by the adapted connection.
\end{abstract}

2000 Mathematics Subject Classification: 53C12, 53D25.

1. Introduction. Let $\left(M, g_{M}\right)$ be a Riemannian manifold. A foliation $\mathscr{F}$ on $M$ is Riemannian and $g_{M}$ bundle-like if all the leaves are locally equi-distant to each other. Such a foliation is characterized by the property that a geodesic orthogonal to the foliation at one point is orthogonal everywhere. For a Riemannian foliation, considerable efforts have been made to give global characterizations of the property that it is harmonic, that is, all of its leaves are minimal submanifolds. For examples, a Riemannian foliation is harmonic if and only if either one of the following conditions holds: (1) it is an extremal of the energy functional for special variations (see [2]); (2) it is an extremal of the energy of the foliation under certain variations of the Riemannian metric of the manifold (see [1]). In this paper, we give a dynamical characterization of the harmonicity of a Riemannian foliation which has the flat normal connection in the sense of Oshikiri [4].

Let $\mathscr{F}$ be a Riemannian foliation of dimension $p$ and codimension $q$ on a Riemannian manifold $M$ of dimension $n(p+q=n)$ with bundle-like metric $g_{M}$. Throughout, we work in the smooth category and the following notations are used:

- $T M$ is the tangent bundle of $M$.

- $L$ and $L^{\perp}$ are the tangent bundle and the normal bundle of $\mathscr{F}$, respectively.

- $\Gamma T M, \Gamma L$, and $\Gamma L^{\perp}$ are the spaces of sections of $T M, L$, and $L^{\perp}$, respectively.

- $\pi: T M \rightarrow L^{\perp}, \pi^{\perp}: T M \rightarrow L$, and $P_{\mathscr{F}}: L^{\perp} \rightarrow M$ are the canonical projections.

- $\nabla^{M}$ is the Levi-Civita connection associated with $g_{M}$.

Since $\mathscr{F}$ is Riemannian, there exists a unique torsion-free metric connection $\nabla$ on $L^{\perp}$ which is called adapted and given as follows (see [2]): for $Z \in \Gamma L^{\perp}$,

$$
\nabla_{X} Z= \begin{cases}\pi[X, Z] & \text { for } X \in \Gamma L, \\ \pi\left(\nabla_{X}^{M} Z\right) & \text { for } X \in \Gamma L^{\perp}\end{cases}
$$

Associated with the above connection there is a bundle map $C_{\mathscr{F}}: T L^{\perp} \rightarrow L^{\perp}$ called the 
connection map associated with $\mathscr{F}$ given as follows. For $\xi \in T_{Z} L^{\perp}$ with $\left(d P_{\mathscr{F}}\right)(\xi) \neq 0$,

$$
C_{\mathscr{F}}(\xi)=\nabla_{\dot{\sigma}(0)} Z,
$$

where $Z$ is a curve in $L^{\perp}$ such that $d /\left.d t\right|_{t=0} Z=\xi$ and $\sigma(t)=P_{\mathscr{F}}(Z(t))$. This map gives a metric $\tilde{g}$ on $L^{\perp}$ defined by

$$
\tilde{g}(\xi, \eta)=g_{M}\left(\left(d P_{\mathscr{F}}\right)_{Z}(\xi),\left(d P_{\mathscr{F}}\right)_{Z}(\eta)\right)+g_{M}\left(C_{\mathscr{F}}(\xi), C_{\mathscr{F}}(\eta)\right)
$$

for $\xi, \eta \in T_{Z} L^{\perp}$. We denote the Riemannian volume form on $L^{\perp}$ associated with $\tilde{g}$ by $\tilde{\mu}$.

We define a local flow $\phi_{t}$ on $L^{\perp}$, called the normal geodesic flow of $\mathscr{F}$ as follows. For $z \in L^{\perp}$, let $\gamma$ be a geodesic with initial velocity $z$. Since $\mathscr{F}$ is Riemannian, $\dot{\gamma}(t) \in L^{\perp}$ for each $t$ in the domain of $\gamma$. We put $\phi_{t}(z)=\dot{\gamma}(t)$ for $z \in L^{\perp}$ and $t$ in the domain of $\gamma$.

A foliation $\mathscr{F}$ is said to have the flat normal connection if the normal bundle $L^{\perp}$ of $\mathscr{F}$ admits an orthonormal frame field $\left\{E_{p+1}, \ldots, E_{n}\right\}$ such that $g_{M}\left(\nabla_{Z}^{M} E_{\alpha}, E_{\beta}\right)=0$ for all $\alpha, \beta=p+1, \ldots, n$ and all $Z \in \Gamma L^{\perp}$.

The purpose of this paper is to prove the following theorem.

THEOREM 1.1. Let F be a Riemannian foliation on a Riemannian manifold which has a flat normal connection and $\tilde{\mu}$ the Riemannian volume form on $L^{\perp}$ corresponding to $\tilde{\mathfrak{g}}$. Then $\mathscr{F}$ is harmonic if and only if $\left(\phi_{t}\right)$ preserves $\tilde{\mu}$.

2. The proof. Let $\zeta$ be a vector field on $L^{\perp}$ generated by the geodesic flow. It suffices to show that $\mathscr{F}$ is harmonic if and only if $\left(\Theta_{\zeta} \tilde{\mu}\right)(z)=0$ at any given point $z \in L^{\perp}$, where $\Theta_{\zeta}$ denotes the Lie derivative. Let $\left\{e_{1}, \ldots, e_{n}\right\}$ be an orthonormal basis of the tangent space of $M$ at the point $m=P_{\mathscr{F}}(z)$ such that $e_{i} \in L_{m}$ for $i=1, \ldots, p$ and $e_{\alpha} \in L_{m}^{\perp}$ for $\alpha=p+1, \ldots, n$. In a neighborhood of $m$, we may choose a frame $\left\{E_{\alpha}: \alpha=p+1, \ldots, n\right\}$ of $L^{\perp}$, called an adapted frame, satisfying the following properties: $E_{\alpha}(m)=e_{\alpha}, \alpha=$ $p+1, \ldots, n, \nabla_{e_{\alpha}} E_{\beta}=\pi\left(\nabla_{e_{\alpha}}^{M} E_{\beta}\right)=0$ and $\nabla_{X} E_{\alpha}=\pi\left(\left[X, E_{\alpha}\right]\right)=0$ for any smooth section $X$ of $L$ on $U$ (see [3]). Since $\mathscr{F}$ has the flat normal connection, we may choose $E_{\alpha}$ so that $\nabla_{E_{\alpha}} E_{\beta}=0$ for $\alpha, \beta=p+1, \ldots, n$. Completing this frame by an orthonormal frame $\left\{E_{i}: i=1, \ldots, p\right\}$ of $L$ with $E_{i}(m)=e_{i}$, we get a local orthonormal frame $\left\{E_{1}, \ldots, E_{n}\right\}$ of $T M$ on a neighborhood $U$ of $m$ with $E_{A}(m)=e_{A}$ for $A=1, \ldots, n$. Let $E_{A}^{H}$ for $A=1, \ldots, n$ be the horizontal lift of $E_{A}$ to $T L^{\perp}$, that is, the unique vector field on a neighborhood of $z$ in $L^{\perp}$ such that $d P_{\mathscr{F}}\left(E_{A}^{H}\right)=E_{A}$ and $C_{\mathscr{F}}\left(E_{A}^{H}\right)=0$, and $E_{\alpha}^{V}$ for $\alpha=p+1, \ldots, n$ the vertical lift of $E_{\alpha}$ on $T L^{\perp}$, that is, the vector field on a neighborhood of $z$ such that $d P\left(E_{\alpha}^{V}\right)=0$ and $C_{\mathscr{F}}\left(E_{\alpha}^{V}\right)=E_{\alpha}$. We put $E_{A}^{H}(z)=e_{A}^{H}$ and $E_{\alpha}^{V}(z)=e_{\alpha}^{V}$. Now we compute

$$
\begin{aligned}
{\left[\left(\Theta_{\zeta} \tilde{\mu}\right)(z)\right]\left(e_{1}^{H}, \ldots, e_{n}^{H}, e_{p+1}^{V}, \ldots, e_{n}^{V}\right) } \\
=-\sum_{i=1}^{p} \tilde{\mu}\left(e_{1}^{H}, \ldots,\left[\zeta, E_{i}^{H}\right](z), \ldots, e_{p}^{H}, e_{p+1}^{H}, \ldots, e_{n}^{H}, e_{p+1}^{V}, \ldots, e_{n}^{V}\right) \\
\quad-\sum_{\alpha=p+1}^{n} \tilde{\mu}\left(e_{1}^{H}, \ldots, e_{p}^{H}, e_{p+1}^{H}, \ldots,\left[\zeta, E_{\alpha}^{H}\right](z), \ldots, e_{n}^{H}, e_{p+1}^{V}, \ldots, e_{n}^{V}\right) \\
\quad-\sum_{\alpha=p+1}^{n} \tilde{\mu}\left(e_{1}^{H}, \ldots, e_{n}^{H}, e_{p+1}^{V}, \ldots,\left[\zeta, E_{\alpha}^{V}\right](z), \ldots, e_{n}^{V}\right) .
\end{aligned}
$$


But,

$$
\begin{gathered}
\tilde{\mu}\left(e_{1}^{H}, \ldots,\left[\zeta, E_{i}^{H}\right](z), \ldots, e_{p}^{H}, e_{p+1}^{H}, \ldots, e_{n}^{H}, e_{p+1}^{V}, \ldots, e_{n}^{V}\right) \\
=\tilde{g}\left(\left[\zeta, E_{i}^{H}\right](z), e_{i}^{H}\right)=g_{M}\left(\left(d P_{\mathscr{F}}\right)\left[\zeta, E_{i}^{H}\right](m), e_{i}\right), \\
\tilde{\mu}\left(e_{1}^{H}, \ldots, e_{p}^{H}, e_{p+1}^{H}, \ldots,\left[\zeta, E_{\alpha}^{H}\right](z), \ldots, e_{n}^{H}, e_{p+1}^{V}, \ldots, e_{n}^{V}\right) \\
=g_{M}\left(\left(d P_{\mathscr{F}}\right)\left(\left[\zeta, E_{\alpha}^{H}\right](z)\right), e_{\alpha}\right),
\end{gathered}
$$

where $m=P_{\mathscr{F}}(z)$ and $\alpha$ is the second fundamental form of $\mathscr{F}$ (see [2]).

Let $W_{i}$ be any vector field on $M$ satisfying $W_{i}\left(\varphi_{t}^{i} m\right)=\tilde{\varphi}_{t}^{i} z$ for the local flows $\left(\varphi_{t}^{i}\right)$ of $E_{i}$ and $\left(\tilde{\varphi}_{t}^{i}\right)$ of $E_{i}^{H}$. From $d P_{\mathscr{F}} \circ E_{i}^{H}=E_{i} \circ P_{\mathscr{F}}$, we have $P_{\mathscr{F}} \circ \tilde{\varphi}_{t}^{i}=\varphi_{t}^{i} \circ P_{\mathscr{F}}$ for any $t$. Therefore,

$$
\begin{aligned}
d P_{\mathscr{F}}\left(\left[\zeta, E_{i}^{H}\right](z)\right) & =\left.\frac{d}{d t}\right|_{t=0}\left(d P_{\mathscr{F}} \circ d \tilde{\varphi}_{-t}^{i}\right)\left(\zeta\left(\tilde{\varphi}_{t}^{i}(z)\right)\right) \\
& =\left.\frac{d}{d t}\right|_{t=0}\left(d \varphi_{-t}^{i} \circ d P_{\mathscr{F}}\right)\left(\zeta\left(\tilde{\varphi}_{t}^{i}(z)\right)\right) \\
& =\left.\frac{d}{d t}\right|_{t=0}\left(d \varphi_{-t}^{i} \circ \tilde{\varphi}_{t}^{i}\right)(z) \\
& =\left.\frac{d}{d t}\right|_{t=0}\left(d \varphi_{-t}^{i}\right)\left(W_{i}\left(\varphi_{t}^{i}(m)\right)\right) \\
& =\left[W_{i}, E_{i}\right](m) .
\end{aligned}
$$

Hence we have

$$
\begin{aligned}
g_{M}\left(d P_{\mathscr{F}}\left(\left[\zeta, E_{i}^{H}\right](z)\right), E_{i}(z)\right) & =g_{M}\left(\left[W_{i}, E_{i}\right], E_{i}\right)(m) \\
& =g_{M}\left(W_{i}, \nabla_{E_{i}}^{M} E_{i}\right)(m) \\
& =g_{M}\left(W_{i}(m), \alpha\left(E_{i}, E_{i}\right)(m)\right) \\
& =g_{M}\left(z, \alpha\left(E_{i}(m), E_{i}(m)\right)\right) .
\end{aligned}
$$

Thus, we have

$$
\begin{aligned}
-\sum_{i=1}^{p} \tilde{\mu}\left(e_{1}^{H}, \ldots,\right. & {\left.\left[\zeta, E_{i}^{H}\right](z), \ldots, e_{p}^{H}, e_{p+1}^{H}, \ldots, e_{n}^{H}, e_{p+1}^{V}, \ldots, e_{n}^{V}\right) } \\
& =-g_{M}\left(z, \sum_{i=1}^{p} \alpha\left(E_{i}(m), E_{i}(m)\right)\right) \\
& =-g_{M}(z, \tau(m)),
\end{aligned}
$$

where $\tau(m)$ is the mean curvature vector of $\mathscr{F}$ at $m$ (see [2]).

On the other hand, we have

$$
g_{M}\left(\left(d P_{\mathscr{F}}\left[\zeta, E_{\alpha}^{H}\right]\right)(m), e_{\alpha}\right)=g_{M}\left(\left[W_{\alpha}, E_{\alpha}\right](m), e_{\alpha}\right),
$$


where $W_{\alpha}$ is any vector field on $M$ satisfying $W_{\alpha}\left(\varphi_{t}^{\alpha} m\right)=\tilde{\varphi}_{t}^{\alpha} z$ for the local flows $\varphi_{t}^{\alpha}$ of $E_{\alpha}$ and $\tilde{\varphi}_{t}^{\alpha}$ of $E_{\alpha}^{H}, \alpha=p+1, \ldots, n$. Since $W_{\alpha}\left(\varphi_{t}^{\alpha} m\right)$ is an integral curve of $E_{\alpha}^{H}$, we have $\pi\left(\nabla_{E_{\alpha}}^{M} W_{\alpha}\right)=C_{\mathscr{F}}\left(E_{\alpha}^{H}\right)=0$. Moreover, by the choice of $\left\{E_{\alpha}\right\}$, we have $\pi\left(\nabla_{W_{\alpha}}^{M} E_{\alpha}\right)(m)=0$. Therefore,

$$
g_{M}\left(\left(d P_{\mathscr{F}}\left[\zeta, E_{\alpha}^{H}\right]\right)(m), e_{\alpha}\right)=g_{M}\left(\left(\nabla_{W_{\alpha}}^{M} E_{\alpha}\right)(m)-\left(\nabla_{E_{\alpha}}^{M} W_{\alpha}\right)(m), e_{\alpha}\right)=0 .
$$

Thus, to complete the proof, it suffices to show that

$$
\tilde{\mu}\left(e_{1}^{H}, \ldots, e_{n}^{H}, e_{p+1}^{V}, \ldots,\left[\zeta, E_{\alpha}^{V}\right](z), \ldots, e_{n}^{V}\right)=0,
$$

that is,

$$
g_{M}\left(C_{\mathscr{F}}\left(\left[\zeta, E_{\alpha}^{V}\right](z)\right), e_{\alpha}\right)=0 .
$$

For this purpose, we introduce a local coordinate system around a point $z \in L^{\perp}$ as follows: let $\left(x^{A}\right)_{A=1, \ldots, n}: U \rightarrow \mathbb{R}^{n}$ be a distinguished chart on a neighborhood $U$ of $m \in$ $M$. To $z \in P_{\mathscr{F}}^{-1}(U)$ with $P_{\mathscr{F}}(z)=m$, we $\operatorname{assign}\left(x^{1}(m), \ldots, x^{n}(m), z^{p+1}(m), \ldots, z^{n}(m)\right)$ as its coordinates, where $z=\sum_{\alpha=p+1}^{n} z^{\alpha}(m) E_{\alpha}(m)$. Let $\gamma$ be a geodesic orthogonal to the leaves of $\mathscr{F}$ and $\left(x^{A}(t): A=1, \ldots, n\right)$ its local coordinates.

Write

$$
\dot{\gamma}(t)=\sum_{\alpha=p+1}^{n} z^{\alpha}(t) E_{\alpha}(\gamma(t)) .
$$

By the choice of $\left\{E_{\alpha}\right\}$, we get

$$
\frac{d}{d t} z^{\alpha}=0
$$

for $\alpha=p+1, \ldots, n$. Moreover, if we express $E_{\alpha}$ as $E_{\alpha}=\sum_{A=1}^{n} f_{\alpha}^{A}\left(\partial / \partial x^{A}\right)$, where $f_{\alpha}^{A}$ is a smooth function on $U$, we have

$$
\sum_{A=1}^{n}\left(\frac{d}{d t} x^{A}\right) \frac{\partial}{\partial x^{A}}=\dot{\gamma}=\sum_{\alpha=p+1}^{n} z^{\alpha} E_{\alpha}=\sum_{\alpha=p+1}^{n} \sum_{A=1}^{n} z^{\alpha} f_{\alpha}^{A} \frac{\partial}{\partial x^{A}} .
$$

Equations (2.10) and (2.11) imply that $\left(x^{A}(t), z^{\alpha}(t)\right)$ satisfy

$$
\frac{d}{d t} x^{A}=\sum_{\alpha=p+1}^{n} z^{\alpha} f_{\alpha}^{A}, \quad \frac{d}{d t} z^{\alpha}=0 .
$$

It follows that $\zeta$ can be locally expressed as

$$
\zeta=\sum_{\alpha, A} z^{\alpha} f_{\alpha}^{A} \frac{\partial}{\partial x^{A}}
$$

A simple computation using the above expression of $\zeta$ gives

$$
\left[\zeta, E_{\alpha}^{V}\right]=-\sum_{A}\left(f_{\alpha}^{A}+\sum_{\beta} z^{\beta} E_{\alpha}\left(f_{\beta}^{A}\right)\right) \frac{\partial}{\partial x^{A}} .
$$


It is easy to show that for a vector field $\xi=\sum_{A} \xi^{A}\left(\partial / \partial x^{A}\right)+\sum_{\alpha} \tilde{\xi}^{\alpha}\left(\partial / \partial z^{\alpha}\right), C_{\mathscr{F}}(\xi)$ is given by

$$
C_{\mathscr{F}}(\xi)=\sum_{\alpha}\left(\tilde{\xi}^{\alpha}+\sum_{\beta, A} \Gamma_{\beta A}^{\alpha} z^{\beta} \xi^{A}\right) E_{\alpha}
$$

where $z=\sum_{\alpha} z^{\alpha} E_{\alpha}$ and $\nabla_{\partial_{A}} E_{\alpha}=\sum_{\gamma=p+1}^{n} \Gamma_{\alpha A}^{\gamma} E_{\gamma}$. Therefore,

$$
C_{\mathscr{F}}\left(\left[\zeta, E_{\alpha}^{V}\right]\right)=-\sum_{\delta, \sigma, A}\left\{f_{\alpha}^{A}+\sum_{\beta} z^{\beta} E_{\alpha}\left(f_{\beta}^{A}\right)\right\} \Gamma_{\sigma A}^{\delta} z^{\sigma} E_{\delta} .
$$

But $\Gamma_{\sigma A}^{\delta}=0$ on $U$ for $A=1, \ldots, n$ and $\delta, \sigma=p+1, \ldots, n$ by the choice of the frame $\left\{E_{A}\right\}$. Hence $C_{\mathscr{F}}\left(\left[\zeta, E_{\alpha}^{V}\right]\right)=0$ and the proof is complete.

ACKNOWLEDGMents. The present work was supported by the Basic Science Research Institute Program, Ministry of Education, 1995, BSRI-95-1422 and Yonsei University Faculty Grant for 1994.

\section{REFERENCES}

[1] M. D. Hvidsten and P. Tondeur, A characterization of harmonic foliations by variations of the metric, Proc. Amer. Math. Soc. 98 (1986), no. 2, 359-362.

[2] F. W. Kamber and P. Tondeur, Harmonic foliations, Harmonic Maps (New Orleans, La, 1980), Lecture Notes in Math., vol. 949, Springer-Verlag, Berlin, 1982, pp. 87-121.

[3] F. W. Kamber, P. Tondeur, and G. Tóth, Transversal Jacobi fields for harmonic foliations, Michigan Math. J. 34 (1987), no. 2, 261-266.

[4] G. Oshikiri, Some remarks on minimal foliations, Tôhoku Math. J. (2) 39 (1987), no. 2, 223-229.

Hobum Kim: Department of Mathematics, Yonsei University, Seoul 120-749, Korea

E-mail address: kimhb@yonsei .ac. $\mathrm{kr}$ 


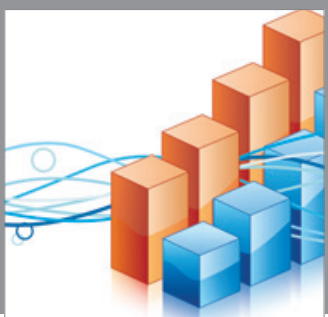

Advances in

Operations Research

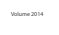

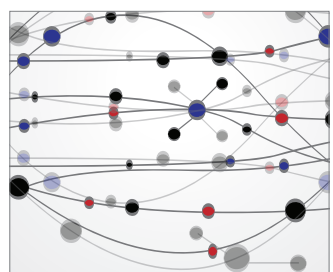

\section{The Scientific} World Journal
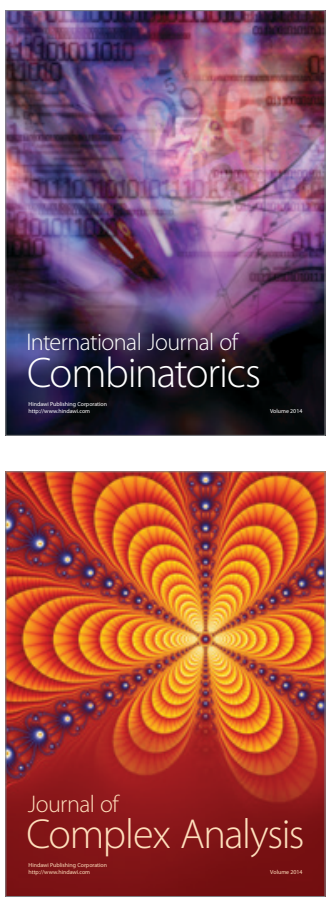

International Journal of

Mathematics and

Mathematical

Sciences
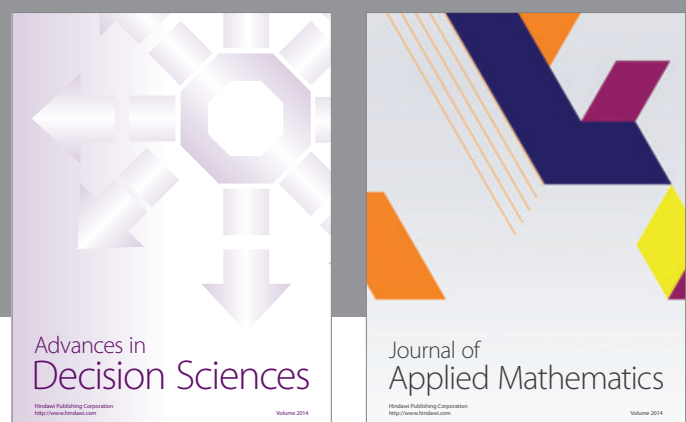

Journal of

Applied Mathematics
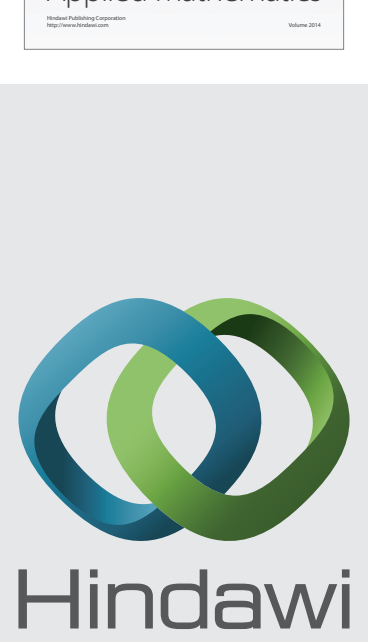

Submit your manuscripts at http://www.hindawi.com
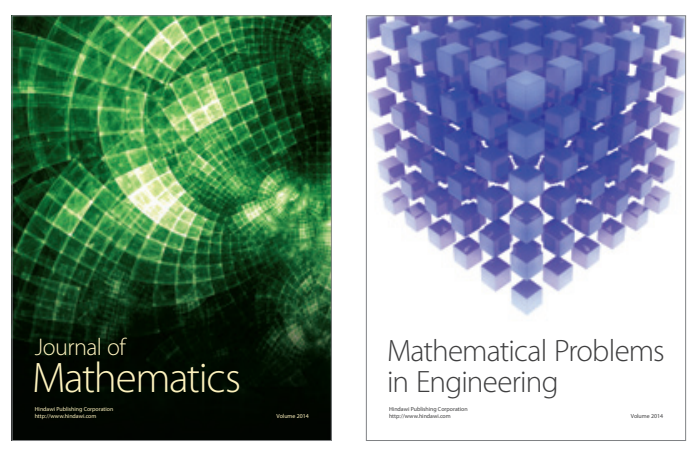

Mathematical Problems in Engineering
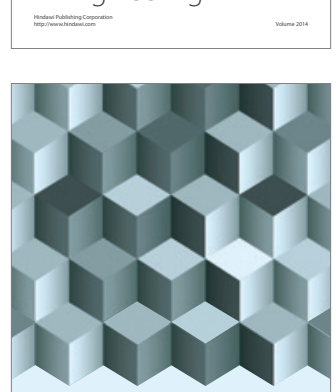

Journal of

Function Spaces
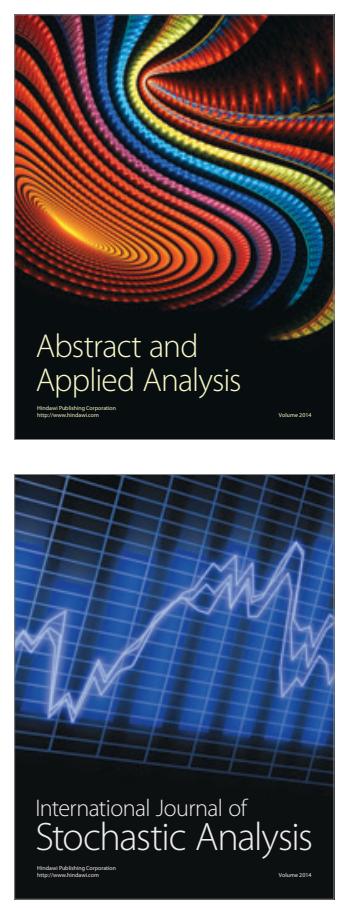

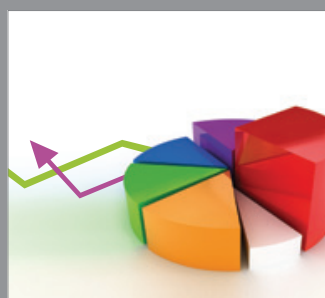

ournal of

Probability and Statistics

Promensencen
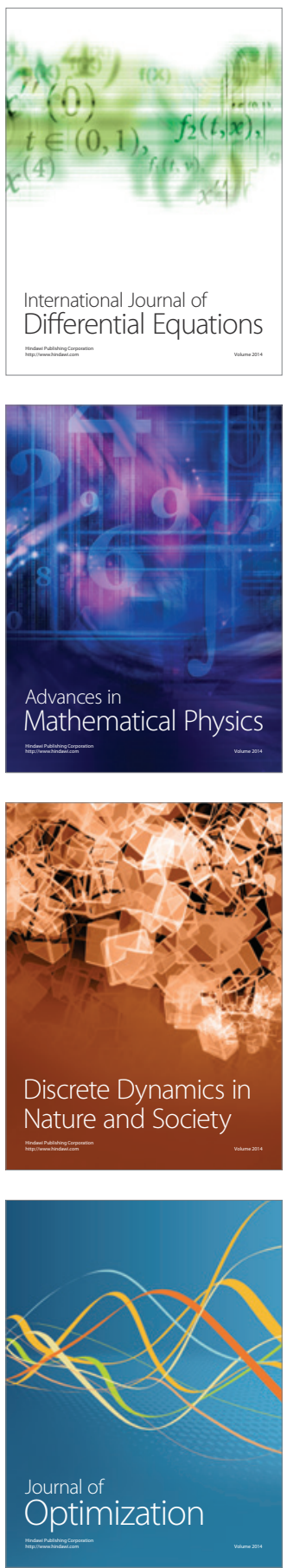